\title{
GEOMAGNETIC JERKS
}

Susan Macmillan

British Geological Survey

Murchison House, West Mains Road, Edinburgh, EH9 3LA, UK.

\section{Observations}

Geomagnetic jerks are abrupt changes in the second time derivative, or secular acceleration, of the magnetic field that arises from sources inside the Earth. They delineate intervals of oppositely signed, near-constant secular acceleration. The first observed geomagnetic jerk was that around 1969 (Courtillot et al, 1978), and since the late $19^{\text {th }}$ century when direct and continuous measurements of the Earth's magnetic field have been made at a number of observatories around the world, geomagnetic jerks have also been observed to occur around 1925, 1978, 1991 and 1999 and possibly also around 1901 and 1913 (e.g. Malin and Hodder, 1982; Courtillot and Le Mouël, 1984; Macmillan, 1996; Alexandrescu et al, 1996; Mandea et al, 2000). These jerks are most readily observed in the first time derivative of the east component at European observatories (Figure 1). Understanding their origin is important, not only because they result from interesting dynamical processes in the core and may help determine the conductivity of the mantle, but also for improving time-dependent models of the geomagnetic field and for the strictly practical purpose of forecasting its future behaviour, for example, as used in navigation. 


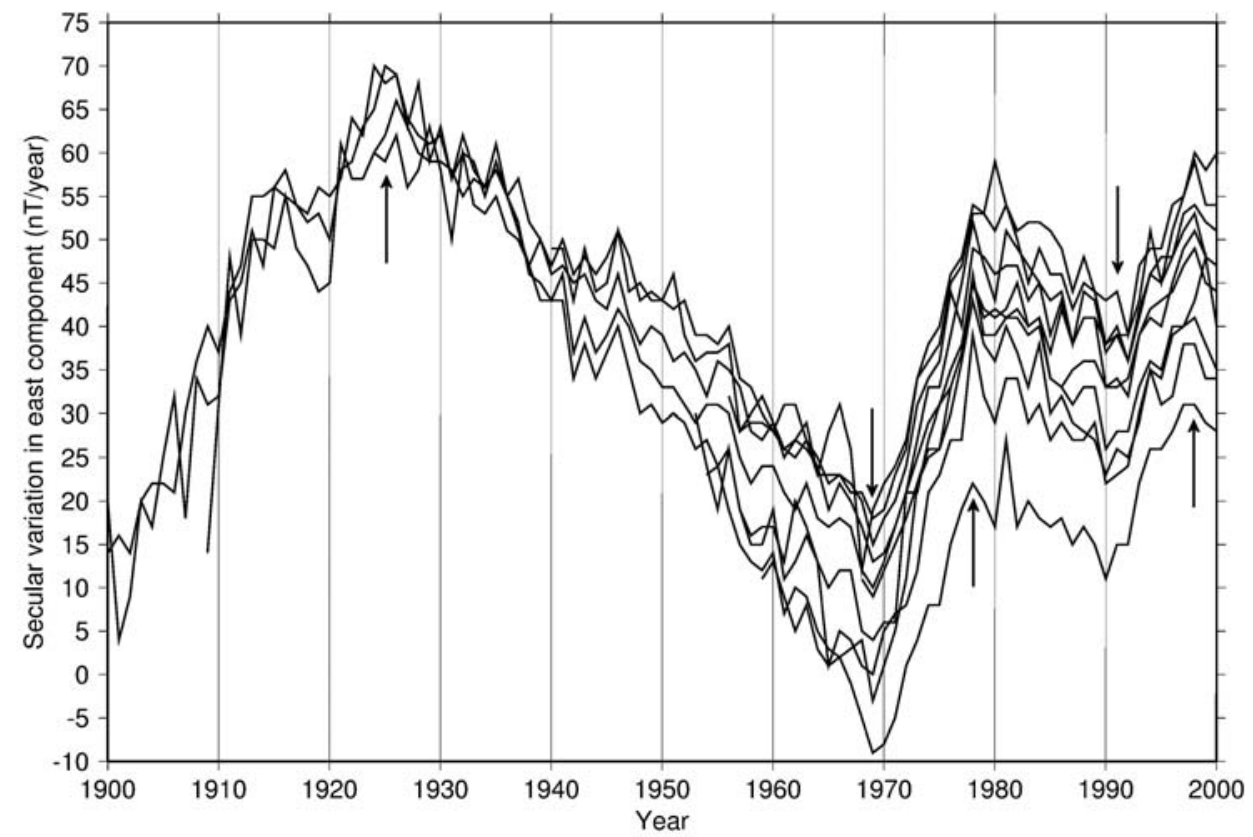

Figure 1 Geomagnetic jerks as seen in the secular variation of the east component of the Earth's magnetic field observed at European observatories. Times of jerks are shown by arrows.

\section{Analysis of jerks}

Many studies have been undertaken to assess jerk characteristics, such as origin, times of occurrence and spatial patterns. At observatories the measured magnetic field is the vector sum of fields arising from three primary sources, namely the main field generated in the Earth's core, the crustal field from local rocks and a combined disturbance field from electrical currents flowing in the upper atmosphere and magnetosphere, which also induce electrical currents in the sea and the ground. As the long-period variations of the disturbance field associated with the 11-year solar cycle are in the same frequency band as variations arising from sources inside the Earth, the separation of the sources is an important part of any analysis of geomagnetic jerks.

Spherical harmonic analysis applied to monthly or yearly mean geomagnetic 
observatory data has shown that jerks are internal in origin and, where sufficient data exist, are global phenomena (e.g. Malin \& Hodder, 1982, McLeod, 1985).

Various analysis techniques have been applied to jerks to investigate specific aspects of their temporal and spatial characteristics. Methods such as optimal piece-wise regression analysis and wavelet analysis have established the exact dates of occurrence at different observatories without any a priori assumptions. Using wavelets, the 1969 and 1978 jerks have been shown to have different arrival times at the Earth's surface, with the northern hemisphere leading the southern hemisphere by about two years (Alexandrescu et al, 1996).

\section{Implications for studies of the Earth's deep interior}

The shortest variations in the Earth's internal field that can be seen at the Earth's surface are determined by the rate of change of the magnetic field at the core-mantle boundary (CMB) and by the electrical conductivity of the mantle through which the magnetic signal from the core must pass. From analyses of surface data we know that the duration of individual jerks, as indicated by the width of impulses in the third time derivative, is no more than about 2 years. However, it is difficult to make any estimate of the rate of change of the magnetic field at the $\mathrm{CMB}$, or the electrical conductivity of the mantle, from geomagnetic field observations at the Earth's surface. As in most geophysical problems assumptions have to be made, or constraints have to be applied using information from other data sources. In the case of geomagnetic jerks one useful independent dataset is Length of Day (LOD) observations. Correlation of the occurrences of jerks with marked changes in the deficit LOD (the former appearing to lead the latter by a few years) indicates some form of coupling between the core and 
mantle. It has recently been shown that inflexions in the time derivative of splines fitted to filtered LOD data from 1960 onwards coincide remarkably well with geomagnetic jerks, including the late arrivals reported for the southern hemisphere (Holme and de Viron, 2005).

Jerks most likely result from dynamic processes inside the Earth that produce changes in the magnetic field near the top of the fluid core. These include the changes caused by advection and shear within the flow. Diffusion is not likely to be an important factor because of the short time-scales involved. Core flows can be estimated directly from observatory secular variation data or indirectly from spherical harmonic models of secular variation. Again, assumptions have to be made or constraints applied so that a unique solution is found. One common assumption applied when determining core flows from magnetic data over time intervals of a few decades is the frozen-flux hypothesis with tangentially geostrophic time-varying flows. With this assumption the fine detail of the secular variation, including jerks, can be well fitted (Jackson, 1997) but the models offer little physical explanation of the flows in terms of core processes. Another assumption that also gives good fits to data is steady flow in a core reference frame that is allowed to rotate about the Earth's rotation axis with respect to the mantle (Holme and Whaler, 2001). This, not surprisingly, provides results which are consistent with LOD data.

A time-dependent flow model that only comprises torsional oscillations, recovers jerks very well while not producing spurious jerks where none are observed (Bloxham et al, 2002). This suggests that the origin of jerks is related to the action of torsional oscillations on the local magnetic field at the CMB. If this is correct, then torsional 
oscillations provide a physical representation for the origin of jerks, for they are not only part of the mathematical representation of core flows but, more importantly, are an actual component of flow predicted to occur in the geodynamo. They also provide the angular momentum changes required to explain the LOD changes. However, the specific details of jerk generation in the core are still to be resolved, in particular the mechanism driving torsional oscillations.

\section{Bibliography}

Alexandrescu, M., Gibert, D., Hulot, G., Le Mouël, J.-L. and Saracco, G., 1996.

Worldwide wavelet analysis of geomagnetic jerks. Journal of Geophysical Research Vol. 101, No. B10, 21,975-21,994.

Bloxham, J., Zatman, S. and Dumberry, M., 2002. The origin of geomagnetic jerks. Nature Vol. 420, 65-68.

Courtillot, V., Ducruix, J., and Le Mouël, J.-L., 1978. Sure une accéleration récente de la variation seculaire du champ magnétique terrestre. Comptes rendus des séances de l’Académie des Sciences Vol. 287, D, 1095-1098.

Courtillot, V. and Le Mouël, J.-L., 1984. Geomagnetic secular variation impulses. Nature Vol. 311, 709-716.

Holme, R. de Viron, O., 2005. Geomagnetic jerks and a high-resolution length-of-day profile for core studies. Geophysical Journal International Vol. 160, 413-413. doi: 10.1111/j.1365-246X.2005.02547.x. Holme, R. and Whaler, K. A., 2001. Steady core flow in an azimuthally drifting reference frame. Geophysical Journal International, Vol. 145, 560-569. Jackson, A., 1997. Time-dependency of tangentially geostrophic core surface motions. Physics of the Earth and Planetary Interiors Vol. 103, 293-312. 
Macmillan, S., 1996. A geomagnetic jerk for the early 1990's. Earth and Planetary

Science Letters Vol. 137, 189-192.

Malin, S. R. C. and Hodder, B. M., 1982. Was the 1970 geomagnetic jerk of internal or external origin? Nature Vol. 296, 726-728.

Mandea, M., Bellanger, E. and Le Mouël, J.-L., 2000. A geomagnetic jerk for the end of the $20^{\text {th }}$ century? Earth and Planetary Science Letters Vol. 183, 369-373.

McLeod, M. G., 1985. On the geomagnetic jerk of 1969. Journal of Geophysical

Research, Vol. 90, B6, 4597-4610.

\section{Cross References (from list at 25.01.2005)}

Decade variations in LOD

Main field modelling

Spherical harmonics

Time-dependent models of the main magnetic field

Torsional oscillations 